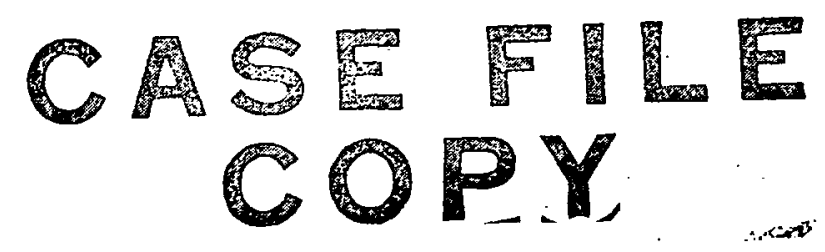

\title{
ON-THE-SHAFT DATA SYSTEMS FOR ROTATING ENGINE COMPONENTS
}

by Daniel J. Lesco, John C. Sturman, and William C. Nieberding

Lewis Research Center

Cleveland, Ohio

TECHNICAL PAPER proposed for presentation at

Symposium on Instrumentation for Airbreathing Propulsion

Monterey, California, September 19-21, 1972 
ON-THE-SHAFT DATA SYSTEMS FOR ROTATING ENGINE COMPONENTS

by Daniel J. Lesco, John C. Sturman, and William C. Nieberding Lewis Research Center

ABSTRACT

Two rotating data systems for engine component testing which demonstrate the techniques of on-the-shaft microelectronic signal conditioning and rotary transformer power- and data-transfer are described. (1) A digital data system provides 69 channels of $1100^{\circ} \mathrm{C}$ maximum thermocouple data with less than 0.5 percent error from a turbine test rig rotating at speeds up to $9000 \mathrm{rpm}$. (2) An analog data system amplifies and transfers 72 channels of dynamic strain data with less than 5 percent error from a compressor rig at speeds above $14000 \mathrm{rpm}$.

\section{INTRODUCTION}

With the advent of smal1, complex, and rugged integrated circuits and the development of a reliable multi-channel rotary transformer, a new solution to the problem of handling large numbers of data channels on rotating engine components is available. As the complexity of new turbines and compressors under development increases, with the corresponding increase in data requirements, the limitations of past methods of transferring measurements across the rotating-to-stationary interface become more evident.

Slip rings have been used extensively to transmit electrical signals from thermocouples or other signal generating transducers. The problems here are that the sliding contacts develop sufficient electrical noise and/or thermal potential to degrade accuracy. Even when the electrical noise problems are not significant, such as when high-level signals are 
being transmitted, slip rings present a maintenance requirement which rapidly becomes more serious with increasing speed.

Radio-frequency transmission of data, usually in the form of FM/FM, has been used successful1y. It is capable of handling many channels by means of time and/or frequency multiplexing. Since $r-f$ transmission, however, requires electrical power on the rotating shaft to operate electronics, the use of a transfer mechanism which can handle both data and power would be a desirable alternate choice.

A number of means have been used to obtain power on a rotating shaft. Batteries can be used to store the energy but have limited power, life, and durability under the high centrifugal accelerations encountered. Slip rings can be used but the maintenance problem is still severe.

As is shown in this paper, a rotary transformer can be used to transfer power to the rotating system and the data from it if the electronic data system on the shaft is capable of accepting ac power and transmitting ac data. This requires sufficient complexity in the data system to convert ac input power into the required dc levels to operate the system and to condition many channels of data into a form suitable for transmission through the rotary transformer.

This paper describes the application of commercially available microelectronics and a rotary transformer to the problem of reliably obtaining many channels of data from a rotating shaft in a maintenance free, accurate manner. Two rotating data systems were developed at Lewis Research Center using the above technique. One system is used to obtain 72 channels of dynamic strain data from a $16000 \mathrm{rpm}$ multi-stage compressor test rig. The second system handles steady state thermocouple data for turbine 
studies.

Although this paper presents details on these two systems as 11lustrations, it is to be emphasized that the basic technique is the important concept to be transferred to the reader. With the rapid advances in electronics, exact duplication of circuitry which is reported a few years after initial development will of ten fail to take advantage of more recent improvements. Also, system size and configuration requirements may necessitate special tailoring for new applications.

The results to be demonstrated herein, therefore, include the system compactness, ruggedness, reliability, and accuracy.

\section{DESIGN OF ROTATING DATA SYSTEMS}

One of the ways data can be categorized is in terms of the maximum rate of change of the parameters measured, that is, the frequency response. It is a common practice in instrumentation to label data which has frequency components of less than a few hertz as "steady state" data, and signals with frequency response above a few hertz as "dynamic" data. In practice, the accuracy requirements for steady state data are usually more stringent than the requirements for dynamic data.

The two rotating data systems provide examples of data handling techniques for both rotating dynamic and steady state data. The two systems have the common characteristics of multiplexing many channels into one path, and signal conditioning the data for transfer from the shaft through a rotary transformer. The following sections of the paper will describe the similarities, and the differences, in detail.

In addition to the problem of high centrifugal forces on the electronics, high temperatures and wide temperature ranges must be taken into 
account in typical engine component applications unless elaborate cooling methods are used for the electronics.

For both systems built, temperatures of $70^{\circ} \mathrm{C}$ were allowed for; in operation, the strain gage system reached $65^{\circ} \mathrm{C}$. This range of temperatures is not destructive to most electronics, but it does make low level signal handling more difficult because of amplifier drifts due to temperature.

\section{THERMOCOUPLE DIGITAL DATA SYSTEM}

\section{Electronics}

The rotating data system for thermocouples, which is fully detailed in reference 1 , is designed to meet the requirements for a specific test function at Lewis Research Center - that of obtaining 60 channels of $1100^{\circ} \mathrm{C}$ full scale thermocouple data from the turbine during blade cooling studies on a modified $\mathrm{J}-75$ test engine operating up to $9000 \mathrm{rpm}$. Its mechanical configuration is designed so that it can replace an existing slip ring assembly with no major modification of the engine.

A simplified block diagram of the system is shown in figure 1, and the system functions as follows. The multiplexer sequentially scans a large number of analog data signals at a rate of 150 channels/sec, connecting one data signal at a time to the data amplifier. This multiplexing of the input signals is an efficient method for processing large numbers of data channels, since only one signal path is then necessary through the amplification and digital conversion functions of the system. The data amplification matches the input signal voltage level to the proper magnitude for conversion to a digital signal. More than one amplifier or amplifier gain changing is required if the data system must handle 
input signals with different full-scale voltages. The analog-to-digital converter transforms the input voltage to an 8-binary-bit digital word proportional to the input. The digital information of each conversion is generated serially ( 1 bit at a time) on a single output line in a form ready for transmission from the rotating shaft. It is very advantageous to digitize the analog amplifier output because subsequent transmission of data is virtually error-free. This data transfer off the shaft and the transfer of power onto the shaft are accomplished with a set of rotary transformer windings. External power is supplied to the rotating electronics in the form of 10-kilohertz square waves applied to the primaries of the power windings. Electronics on the shaft rectify, filter, and regulate the transformer secondary voltage to provide the dc voltage supply levels necessary for operation of the system. The 10-kilohertz power input is also used (counted down in frequency) as a timing (clock) signal by the A/D converter.

The philosophy for the entire system design was to use commercially available integrated circuits (IC) wherever possible. Due to the extensive selection of integrated circuits available, the multiplexer and A/D converter are comprised almost exclusively of digital integrated circuits.

The data system is designed to process differential input signals, such as those provided by thermocouples.. This specification requires that both the high and low sides of a differential input signal be switched by the multiplexer. For the 72 channels of the data system, a total of 144 input switches are needed.

The semiconductor switches used are metal-oxide-silicon-field-effect- 
transistor (MOSFET) switches packaged in groups of six per integrated circuit. These MOSFET switches have the ideal switch characteristic of zero offset voltage, but are limited in some applications by slow switching speed and high "on" resistance. The "on" resistance is about 300 ohms, while the "off" resistance is of the order of $10^{11}$ ohms. The switch turnon and turnoff times are about 2 microseconds.

Figure 2 shows one side of a two-sided printed circuit board which holds the switches and associated logic for 36 differential channels of data. Input connections to the switches are made to the solder pads around the circumference of the board.

The desired full scale of $1100^{\circ} \mathrm{C}$ for Chromel-Alumel thermocouples requires a $50 \mathrm{mV}$ full scale input range for the data system. Since the A/D converter provides a full scale digital output of 8 bits (decimal equivalent of 255) for a 5 volt input, an amplifier gain of 100 is used. The amplifier offset drift characteristic of less than $1 \mu \mathrm{V} /{ }^{\circ} \mathrm{C}$ contributes negligible errors over the design temperature range.

With a resolution of 1 part in 255, and a full scale of approximately $1100^{\circ} \mathrm{C}$, the 1 count resolution of the data system is about $5^{\circ} \mathrm{C}$. Calibration voltages are internally generated in the data system to provide a check on the system operation. Two of the data channels are used for calibration signals. For channel 1, an input voltage proportional to the A/D converter reference supply is generated by a simple resistor divider. The channel 1 digital output provides a monitor primarily on the data amplifier gain. Channel 2 is connected to a calibration voltage generated independently of the converter reference by a separate temperature-compensated zener diode and a divider network. This 
calibration signal provides information primarily on the stability of the system reference voltage.

A third check on the system operation is provided during the multiplexer reset time. During this time, the data amplifier inputs are shorted to ground through MOSFET switches and the A/D converter output is a measurement of negative-polarity data amplifier offset voltage:

Although the information obtained from the three calibration signals does not provide independent information on amplifier gain, offset, or reference voltage, the combination can be used to interpret most discrepancies which could occur in the system operation.

The rotary transformer used in this system consists of four separate, isolated transformers a11 mounted in a common housing. Each of the four nonrotating stator windings is magnetically coupled to its corresponding rotor winding in such a fashion that transformer operation is not influenced by rotation. A11 transformers have a 1 to 1 turns ratio and are shielded from each other to prevent cross coupling. Three of the rotary transformer windings feed power to the data system as 20-volt-peak square waves. These are rectified, filtered, and regulated by zener diodes to provide the necessary voltages for the system. Zener regulation was chosen for simplicity and is adequate since none of the basic supply voltages require close regulation. The fourth transformer winding is used to transfer the serial digital data from the system to the readout electronics. An emitter follower stage is used to buffer the output from the $A / D$ converter and drive the transformer. The result is approximately 5-volt pulses from the rotary transformer stator. 
Mechanical Design

A logical configuration for a rotating system is a round package. Therefore, this system was built using round, double-sided printed circuit boards with plated-through holes. Components considered to be most sensitive to acceleration were mounted near the center of the board when possible. All interboard connections were made near the periphery of the boards, which facilitated testing of the assembled system. The boards are heavily coated with a clear epoxy to hold a11 components securely to the board.

The complete system is assembled by stacking the circuit boards together with spacers and making the required interconnections. Figure 3 shows this stage in the construction of the engine system. The five-board module, which is 9 centimeters in diameter by 6 centimeters long, mates at one end to a circular terminal ring which is the interface between the data system and the thermocouples from the engine. The terminal ring also serves as the cold junction for the thermocouples, and its temperature is monitored by a thermistor cemented to it. One input channel is used to read out this thermistor signal.

Rigid mounting of the complete electrical assembly is accomplished by clamping it to the inner rotating support plate with three tie rods. A metal spacer is used at the opposite end for alinement so that any small irregularity in the boards will not affect the mechanical mounting. The complete system showing the wired electronic assembly and partial housing is shown in figure 4. Connections to the rotary transformer are made at the end of the package through the hollow shaft and to an eight-pin terminal ring. Use of the two terminal rings, accessible through a slotted 
housing, makes it possible to connect or disconnect the data system in a minimum amount of time. The housing shown in figure 4 is considerably larger than necessary to properly support the electronics package. Its dimensions were dictated by the practical requirement that it be identical in size to a slip ring assembly which it replaces.

Following application of epoxy after the mounting of components on the boards, each board was spun (nonoperating) to $15000 \mathrm{rpm}$ and then tested electrically. After the entire package was assembled, a cold spin rig test of several hours at $9000 \mathrm{rpm}$ was performed. During this test, millivolt input signals simulating thermocouple inputs were applied through slip rings to the data system. The system demonstrated less than 0.5 percent error for all tests.

Once the data has been transferred from the shaft, a considerable amount of electronics is required to handle the serial wavetrain of digital data - that is, to group it into 8-bit data words and synchronize word with data channel. If sufficient transformer windings are avai1able, synchronization signals could also be transferred from the shaft. In this example, nonrotating electronics are used to examine the data wavetrain for a synchronization code related to the calibration channels and to "lock" onto this pattern. Once synchronized, the data is fed both to a central recording facility and to a bar graph type display. Although the electronics required is complex, it can be implemented with standard equipment and standard engineering design.

Figure 5 shows the entire system mounted on the J-75 engine during checkout. At the time of writing, engine data had not yet been obtained. 


\section{DYNAMIC STRAIN GAGE ROTATTNG DATA SYSTEM}

This system was designed to obtain dynamic strain data from a multistage compressor test rig at Lewis Research Center. The requirements were to measure 60 channels of dynamic strain of up to $2000 \mu$ strain over a frequency range of 150 to $3000 \mathrm{~Hz}$, to about 5 percent accuracy at $16000 \mathrm{rpm}$.

A block diagram of the system is shown in Figure 6 . This system is somewhat less complex than the digital rotating data system. As in the other system, however, the data channels are interrogated one at a time. The maximum rate for this system is 80 channels/sec.

A considerable amount of on-the-shaft power is saved by applying the constant current supply only to that strain gage which is connected to the data amplifier. The value of current used is $5 \mathrm{~mA}$. Another advantage is the elimination of crosstalk problems, since only one strain gage is powered at any time.

The amplifier is AC coupled to the multiplexer output to allow the amplification of only the dynamic strain data. In order to amplify data down to a few hundred hertz, the amplifier input time constant must be on the order of $10 \mathrm{msec}$. However, in the event of failure in a gage, either open or short, the coupling capacitor will be subjected to a transient of several volts and must recover sufficiently to levels less than a millivolt in time for the next channel to be accurately read. This requires a short time constant settling time at the beginning of each switch transition. To accomplish this, a semiconductor switch is closed in parallel with the amplifier input resistor for a period of about a millisecond. The effective RC time constant is reduced by approximately 1000 , to a 
value of about $10 \mu \mathrm{sec}$. After the $1 \mathrm{msec}$ settling time, the capacitor has reached its equilibrium DC voltage.

The scan rate of the multiplexer in this system can be externally controlled, including provision for stopping the multiplexer on any chosen channel for continuous data. A separate synchronization pulse output winding is also provided. Figure 7 shows a representative timing diagram of the data, sync, and scan control windings.

Power is $\mathrm{AC}$ coupled at $20 \mathrm{KHz}$ into this system with rectification and regulation again providing the DC voltages for operation of the electronics. The higher power frequency was used to further remove potential noise from data frequencies of interestiso that output filtering could be used.

External electronics, of course, are required for control and readout of the data system. The primary display instrument is an oscilloscope, with tape recording as a means of permanent recording.

Figure 8 is a photograph of the completely assembled data system. Connections are made to the strain gages through an 85-pin high density connector at the end of the shaft at the right. This allows for easy removal of the system from the rig.

The complete system was tested at $20000 \mathrm{rpm}$ for several hours in a turbine-powered spin rig. It has successfully been gathering compressor study data for many hours at speeds up to $15000 \mathrm{rpm}$. The only electronics-responsible failures experienced were the cutting of two multiplexer input wires due to centrifugal action and excessive slack. The wires were readily spliced with no recurrence. 
Figure 9 is a photograph of the rotating data system after incurring mechanical damage due to excessive coupling shaft axial movement. This occurrence provided an example of the ruggedness of the electronics. Shaft movement away from the printed circuit boards resulted in the breaking at the boards of all 144 multiplexer input leads coming from the connector. The shredded remains of the leads can be seen adhering to the insides of the housing struts. No damage was incurred by any of the boards. Upon replacement of the damaged mechanical parts and rewiring of the inputs, the system performed without fault.

\section{ROTATING PRESSURE MEASUREMENTS}

In addition to temperature and strain measurements, rotating pressure measurements are important to turbine development. A modified digital rotating data system. has been developed for handling $\pm 100 \mathrm{mV}$ full scale data from ten variable capacitance pressure transducers, in addition to data from 60 thermocouples. This requires the use of a second amplifier switched into the data path for the 10 pressure channels. Increased power handling capabilities are also required for the data system to power the ten transducers. This system has not yet been tested with the pressure transducers.

\section{DISCUSSION}

It has been demonstrated that rotating data systems using microelectronics and rotary transformers can reliably provide large amounts of accurate data from rotating engine components under test.

A few of the system limitations should be reviewed.

1. A limitation of a time division multiplexer is that time correlation for data at frequencies above a few hertz is not possible. 
2. A failure in a critical system component, such as the amplifier or A/D converter, will result in the loss of all data, whereas parallel transmission of transducer signals directly through slip rings to external electronics is probably more fail safe from a total system point of view.

3. Losing direct contact with the transducers limits the analyzing of transducer failures.

4. Although the initial design is quite flexible, once the system is built, changes (i.e., number of channels, amplifier gain, number of amp1ifiers) are difficult unless sufficient complexity was initially planned for to allow variation of characteristics.

For complex rotating data systems, especially those handling more than one type of transducer, there is a distinct advantage in having a minicomputer to handle and display the data as opposed to designing special purpose electronic hardware. Although some of the burden falls to the computer programmer, significant manpower can be saved by taking advantage of the data handing and manipulating capabilities of the minicomputer.

\section{REFERENCES}

1. Lesco, Daniel J.; Sturman, John C.; and Nieberding, William C.: Rotating Shaft-Mounted Microelectronic Data System. NASA TN D-5678, 1970 . 


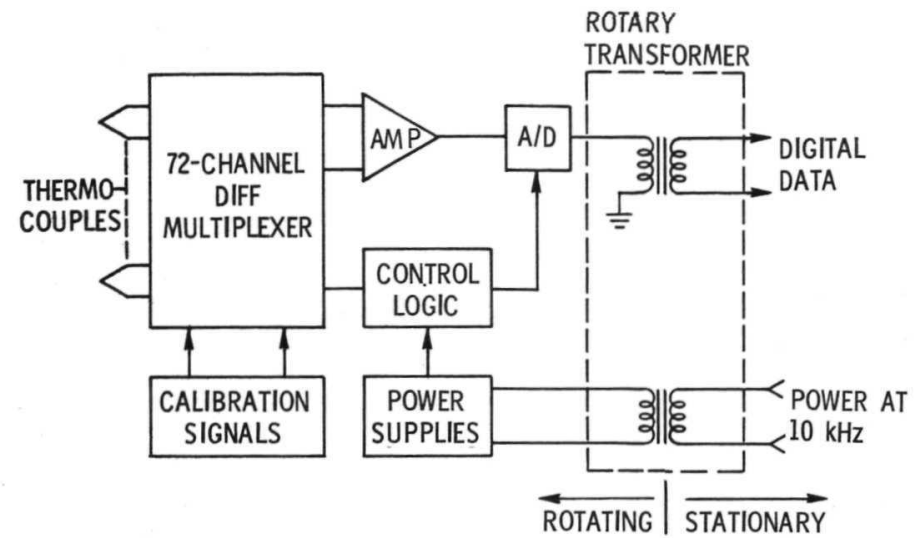

Figure 1. - Therm ocouple data system block diagram.

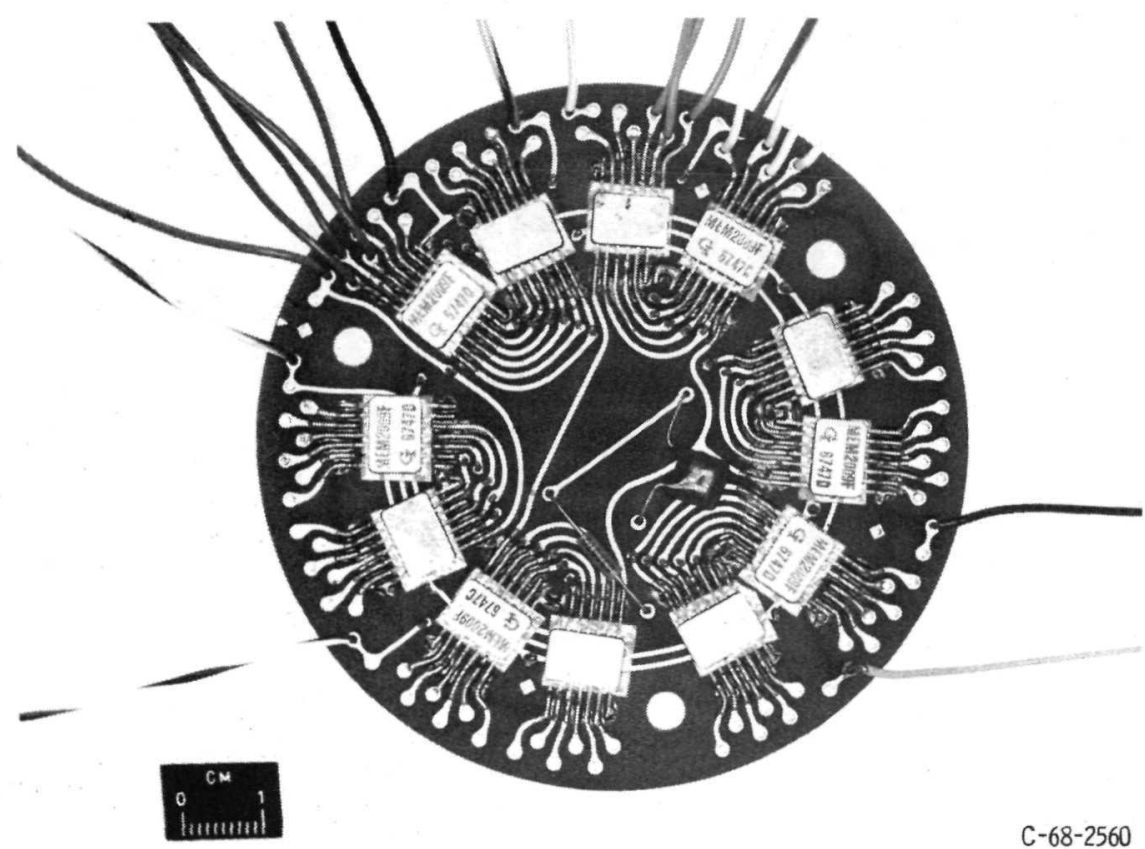

Figure 2. - 36-Channel multiplexer. 


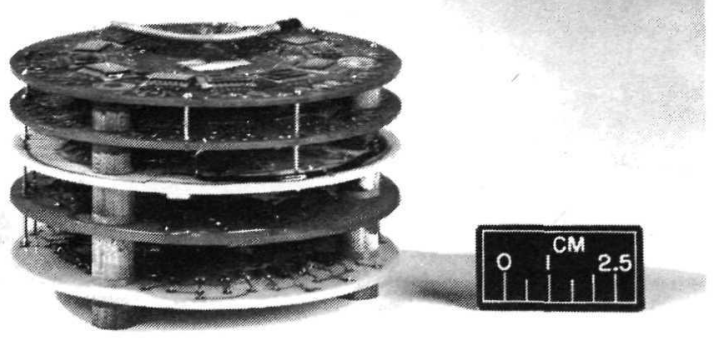

C- $69-1835$

Figure 3. - Assembled electronics package.

ñ

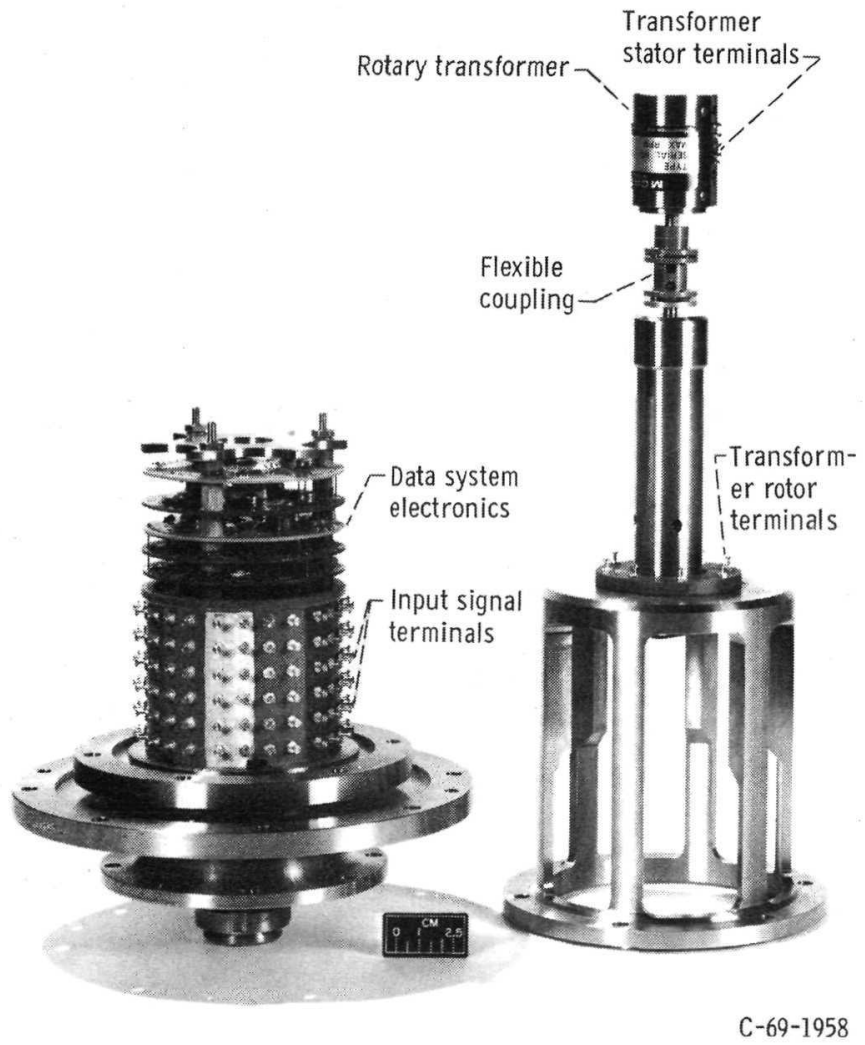

Figure 4. - Engine data system and housing. 


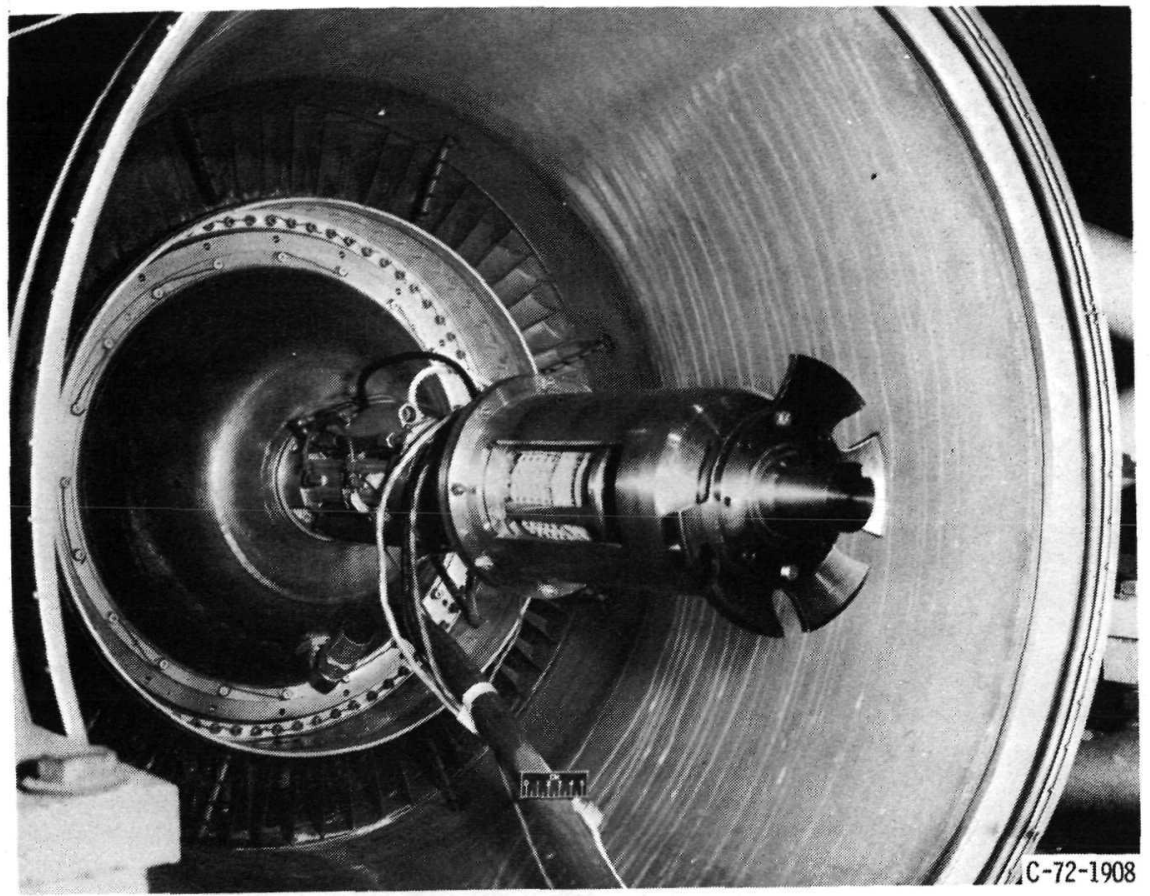

Figure 5. - Rotating data system on J-75 engine.

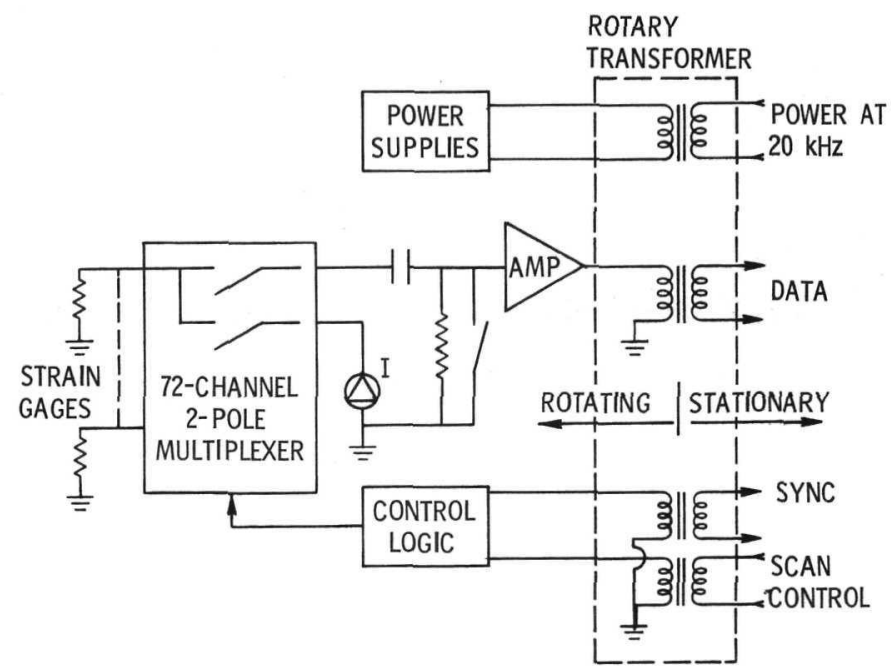

Figure 6. - Strain gage data system block diagram. 


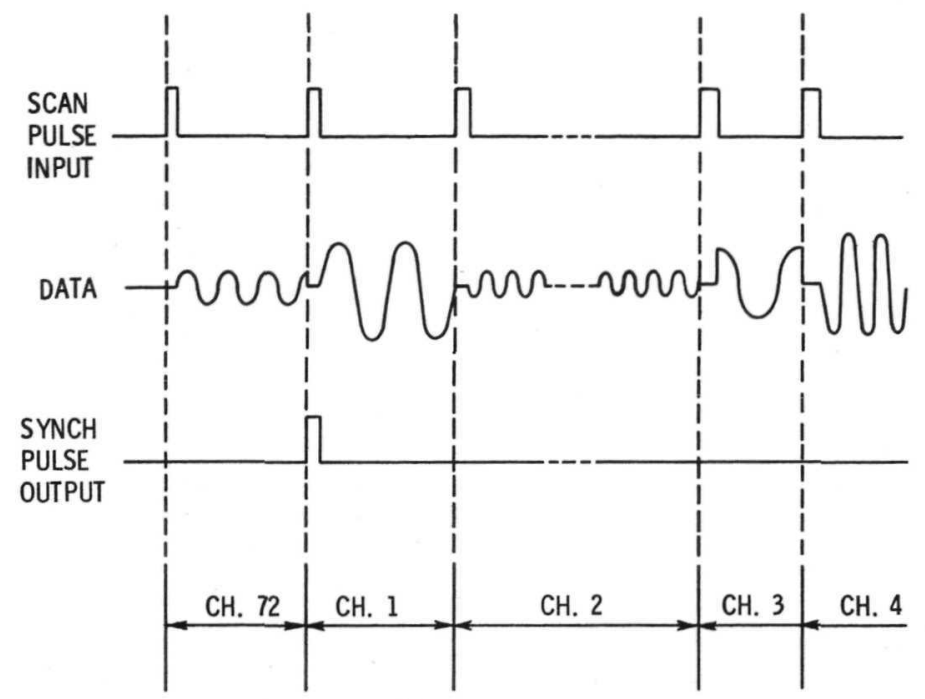

Figure 7. - Strain gage data system waveforms.

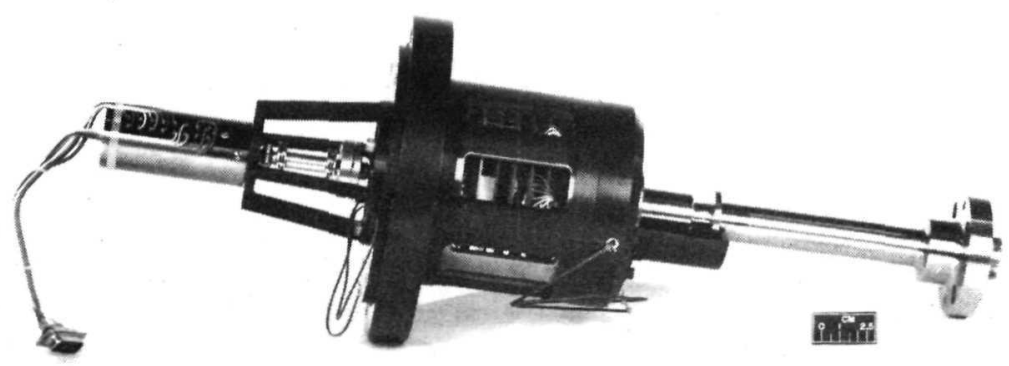

Figure 8. - Strain gage data system. 
$m$
in
0
1
1
1

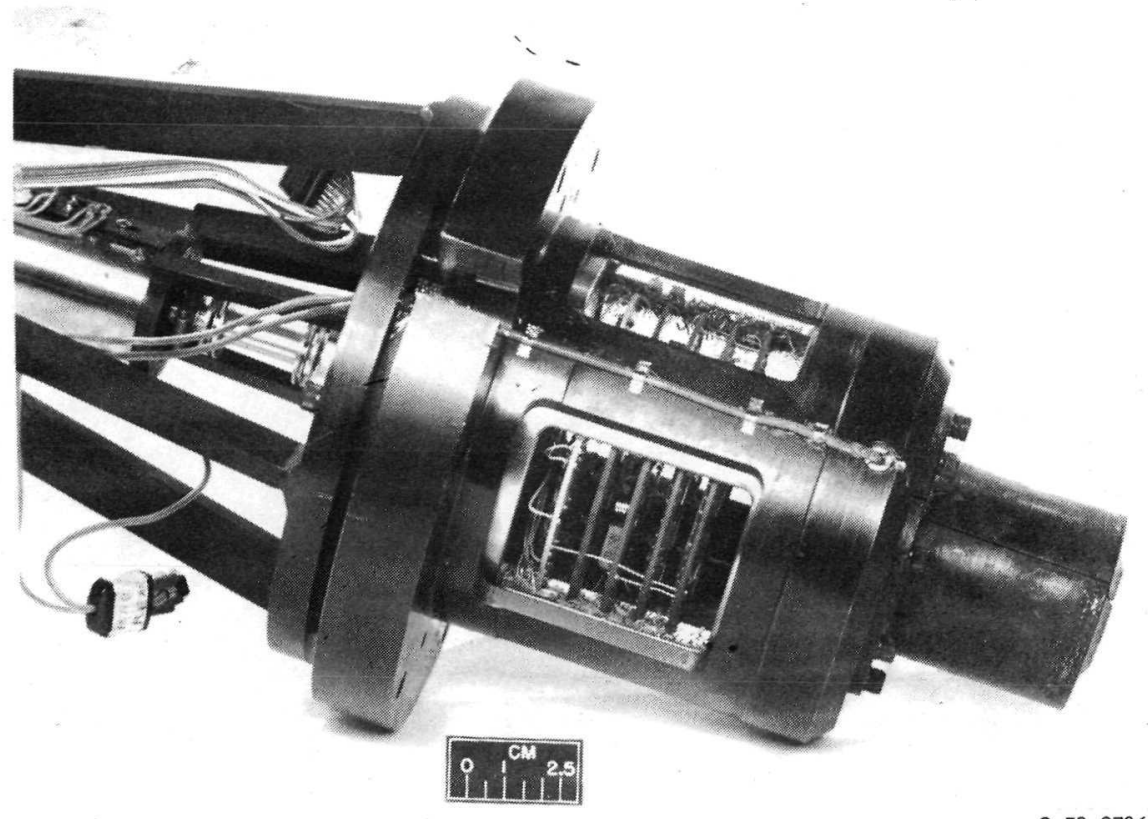

Figure 9. - Damaged data system. 